\title{
INTERCUSP GEODESICS AND THE INVARIANT TRACE FIELD OF HYPERBOLIC 3-MANIFOLDS
}

\author{
WALTER D. NEUMANN AND ANASTASIIA TSVIETKOVA
}

(Communicated by Martin Scharlemann)

\begin{abstract}
Given a cusped hyperbolic 3-manifold with finite volume, we define two types of complex parameters which capture geometric information about the preimages of geodesic arcs traveling between cusp cross-sections. We prove that these parameters are elements of the invariant trace field of the manifold, providing a connection between the intrinsic geometry of a 3manifold and its number-theoretic invariants. Further, we explore the question of choosing a minimal collection of arcs and associated parameters to generate the field. We prove that for a tunnel number $k$ manifold it is enough to choose $3 k$ specific parameters. For many hyperbolic link complements, this approach allows one to compute the field from a link diagram. We also give examples of infinite families of links where a single parameter can be chosen to generate the field, and the polynomial for it can be constructed from the link diagram as well.
\end{abstract}

\section{INTRODUCTION}

The invariant trace field is one of the most used tools in the study of hyperbolic manifolds from the number-theoretical point of view. In this note, we discuss how this arithmetic invariant is related to the intrinsic geometry of the manifold, and to intercusp geodesics in particular. This geometric perspective allows one to compute the invariant trace field of many hyperbolic link complements from their diagrams.

$X$ will always denote a complete orientable hyperbolic 3-manifold of finite volume. If $\Gamma$ is the image of a discrete faithful representation of the fundamental group of $X$ into $\operatorname{Isom}^{+}\left(\mathbb{H}^{3}\right)$, then $X$ can be regarded as the quotient $\mathbb{H}^{3} / \Gamma$. After picking upper half space coordinates $\mathbb{C} \times \mathbb{R}_{+}$on $\mathbb{H}^{3}$ we can identify $\mathrm{Isom}^{+}\left(\mathbb{H}^{3}\right)$ with $\operatorname{PSL}_{2}(\mathbb{C})$ acting so that its action on the sphere at infinity $\mathbb{C} \cup\{\infty\}$ is by Möbius transformations. This identification is only determined up to conjugacy, since it depends on the coordinate choice. Nevertheless, for an element $\gamma \in \Gamma$ we can speak of the trace $\operatorname{tr}(\gamma)$ (determined only up to sign), since the trace of a matrix is invariant under conjugacy.

The field $\operatorname{tr}(\Gamma)$ generated over $\mathbb{Q}$ by the traces of elements of $\Gamma$ is called the trace field of $X$. In view of the Mostow-Prasad rigidity, $\operatorname{tr}(\Gamma)$ is a finite extension of $\mathbb{Q}$ (the proof can be found in [7]). It is an invariant of the group $\Gamma$ and thus is a topological invariant of the manifold, but in general it is not an invariant of its commensurability class in $\mathrm{PSL}_{2}(\mathbb{C})$ (see [2,10,12, for counterexamples).

Received by the editors October 10, 2014 and, in revised form, December 25, 2014.

2010 Mathematics Subject Classification. Primary 57M25, 57M50, 57M27.

Key words and phrases. Link complement, hyperbolic 3-manifold, invariant trace field, cusp, arithmetic invariants. 
Consider the subgroup $\Gamma^{2}=\left\langle\gamma^{2} \mid \gamma \in \Gamma\right\rangle$ of $\Gamma$. The invariant trace field is the field generated over $\mathbb{Q}$ by the traces of $\Gamma^{2}$. Often denoted by $k(\Gamma)$ or $k(X)$, the invariant trace field is a topological and commensurability invariant of the manifold (12]). Clearly, it is a subfield of the trace field. If $X$ is a link complement, it actually coincides with the trace field (this was proven in [12] for knots, and in [10] for links).

In [10, it is shown that the invariant trace field contains useful geometric information about the hyperbolic manifold. In particular, if $\Gamma$ contains parabolic elements, the invariant trace field is equal to the field generated by shapes of all tetrahedra of any ideal triangulation of $X$ (by "shape" of an ideal tetrahedron we mean the cross-ratio of the vertices as elements of $\mathbb{C} \cup\{\infty\}$; it is determined up to a three-fold ambiguity, depending on an orientation-compatible choice of ordering of the vertices). Here we show that the invariant trace field also contains certain complex "intercusp parameters" that measure distances and angles between cusps (more precisely, between preimages in $\mathbb{H}^{3}$ of cusp cross-sections), as well as "translation parameters" which measure displacement between ends of intercusp geodesic arcs.

With the ideas described in 14 this enables one to give exact computations of generators of the invariant trace field of a hyperbolic link directly from a link diagram in many cases. For example, for 2-bridge links, we demonstrate that a single intercusp parameter suffices, with a polynomial which can be constructed combinatorially from the diagram. Previously known methods included finding decimal approximations of simplex shapes, and then making an intelligent guess of the corresponding polynomial for the field using the LLL algorithm (see [3]).

\section{THE PARAMETERS}

In this section, we assume our hyperbolic 3-manifold $X$ has at least one cusp. We introduce a complex parameter that captures geometric information about distances and angles between preimages of cusp cross-sections in $X$. Later we will use it to compute invariant trace fields of links from their diagrams. The idea of such a parameter appeared in [14] for intercusp geodesics that correspond to crossings of a link diagram. Here we will consider it in a more general setting. Our definition also dovetails with a notion of "complex length" of a geodesic that was introduced in [10].

We will speak loosely of the sphere at infinity as the "boundary" of $\mathbb{H}^{3}$. Each horospherical cusp cross-section of $X$ is a torus which lifts to a set of horospheres tangent to the boundary of $\mathbb{H}^{3}$. The point of tangency of such a horosphere $H_{i}$ will be referred to as the center of $H_{i}$ and will be denoted by $P_{i}$.

For each cusp of $X$ we choose an essential simple closed curve in a horospherical section of the cusp, which we call the meridian. (If $X$ is the complement of an oriented link in $S^{3}$, we choose the standard meridians, which are oriented using the right hand screw rule.) Henceforth we will assume that the horospherical torus cross-section of each cusp of $X$ has been chosen so that the (geodesic) meridian curve on this torus has length 1 . Such choice guarantees that the horoballs have disjoint interiors. The horoballs are in fact disjoint in every case except for the figure-eight knot complement in $S^{3}$ (as was proved in [1]). For the figure-eight, the corresponding cross-sectional torus touches itself in two points.

In the following, we only consider horospheres $H_{i}$ in $\mathbb{H}^{3}$ which are lifts of cusp cross-sections of $X$ as above. We call these marked horospheres, since each such 
horosphere can be regarded as the complex plane, with coordinates in this plane specified (up to translation) by declaring that the meridional translation corresponds to the real number one. If we position $H_{i}$ by an isometry of $\mathbb{H}^{3}$ to be the Euclidean plane $t=1$ centered at $\infty$ (which we denote by $H_{\infty}$ ), then the meridional translation on $H_{i}$ is represented by the matrix $\left(\begin{array}{ll}1 & 1 \\ 0 & 1\end{array}\right)$. For convenience we will often abuse the distinction between $\operatorname{Isom}^{+}\left(\mathbb{H}^{3}\right)=\mathrm{PSL}_{2}(\mathbb{C})$ and $\mathrm{SL}_{2}(\mathbb{C})$ and simply work with matrices. Since $\mathrm{PSL}_{2}(\mathbb{C})=\mathrm{PGL}_{2}(\mathbb{C})$ we sometimes use matrices in $\mathrm{GL}_{2}(\mathbb{C})$ (but note that the PSL trace $\operatorname{tr}(A)$ of such a matrix $A$ is $\frac{ \pm \operatorname{trace}(A)}{\sqrt{\operatorname{det}(A)}}$ ).

Let $\gamma\left(H_{1}, H_{2}\right)$ be the shortest geodesic arc connecting two marked horospheres $H_{1}$ and $H_{2}$ in $\mathbb{H}^{3}$. If it has length $d$, we can parallel translate along $\gamma\left(H_{1}, H_{2}\right)$ and then rotate by an angle $\theta$ in $H_{2}$ to take the meridional direction on $H_{1}$ to the meridional direction on $\mathrm{H}_{2}$. We call the complex number

$$
\delta\left(H_{1}, H_{2}\right):=d+i \theta
$$

the complex distance between $H_{1}$ and $H_{2}$ and we call

$$
w\left(H_{1}, H_{2}\right):=e^{-\delta\left(H_{1}, H_{2}\right)}
$$

the intercusp parameter 1 Figure 1 illustrates a complex intercusp distance with argument $\theta$ between 0 and $\pi$.

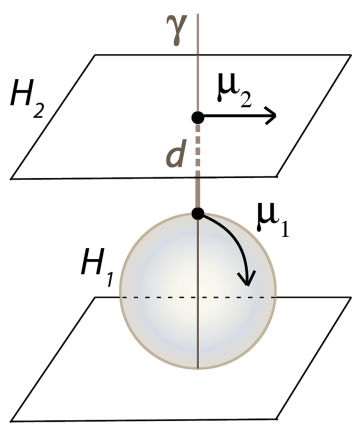

FiguRE 1. Intercusp distance.

Observe that if we position one of $H_{1}$ and $H_{2}$ as $H_{\infty}$ and the other with center at 0 , then the involution given by the matrix

$$
M\left(H_{1}, H_{2}\right):=\left(\begin{array}{cc}
0 & w\left(H_{1}, H_{2}\right) \\
1 & 0
\end{array}\right) \in \operatorname{PGL}(2, \mathbb{C})
$$

exchanges $H_{1}$ and $H_{2}$ taking meridian direction of $H_{1}$ to that of $H_{2}$.

We will also use another complex parameter defined as follows. Suppose we have three marked horospheres $H_{1} \neq H_{2} \neq H_{3}$, and $P_{i}$ is the center of a marked horosphere $H_{i}$ for $i=1,2,3$. Suppose $H_{2}$ intersects the geodesics $P_{1} P_{2}$ and $P_{2} P_{3}$ in points $N$ and $M$ respectively (see Figure 2). Using the affine complex structure on $\mathrm{H}_{2}$ there is a complex number determining a translation mapping $N$ to $M$. We call this complex number the translation parameter $23\left(H_{1}, H_{2}, H_{3}\right)$. Note that

\footnotetext{
${ }^{1}$ In 14 certain intercusp parameters, with opposite sign, are called "crossing labels".

${ }^{2}$ In 14 certain translation parameters, sometimes with sign changed, are called "edge labels".
} 
if we position $H_{2}$ as $H_{\infty}$, then $u\left(H_{1}, H_{2}, H_{3}\right)$ will be the complex number that corresponds to the translation between the centers $P_{1}, P_{3}$ of $H_{1}, H_{3}$. Then the matrix

$$
M\left(H_{1}, H_{2}, H_{3}\right):=\left(\begin{array}{cc}
1 & u\left(H_{1}, H_{2}, H_{3}\right) \\
0 & 1
\end{array}\right)
$$

gives a translation of $H_{2}$ taking $N$ to $M$.

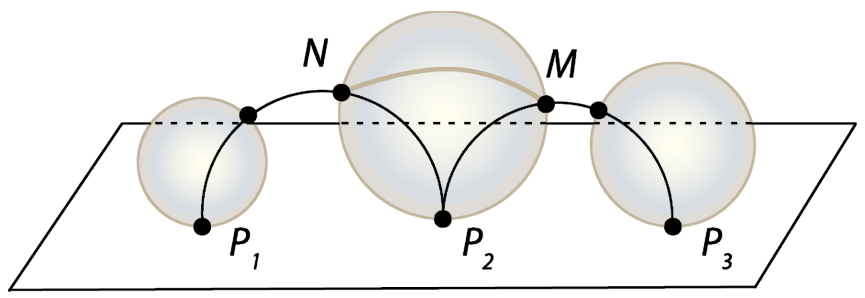

FiguRE 2. Auxiliary parameter.

\section{Properties of the parameters}

We first give versions of Theorems 4.1 and 4.2 of 14 adapted to the orientation conventions of this paper, along with quick proofs of both. Let $H_{1}, \ldots, H_{n}$ be marked horospheres with $H_{i} \neq H_{i+1}$ for $i=1, \ldots, n-1$ and $H_{n} \neq H_{1}$. We use the notation of equations (11) and (2).

Theorem 3.1 (Compare Theorem 4.2 of [14]). With indices taken modulo n,

$$
\prod_{i=1}^{n} M\left(H_{i}, H_{i+1}\right) M\left(H_{i}, H_{i+1}, H_{i+2}\right)=I \quad \text { in } \operatorname{PGL}(2, \mathbb{C}) \text {. }
$$

Theorem 3.2 (Compare Theorem 4.1 of [14]). Recall $P_{i}$ denotes the center of $H_{i}$. The shape parameter of the ideal simplex with vertices $P_{i-1}, P_{i}, P_{i+1}, P_{i+2}$ is

$$
\frac{-w\left(H_{i}, H_{i+1}\right)}{u\left(H_{i-1}, H_{i}, H_{i+1}\right) u\left(H_{i}, H_{i+1}, H_{i+2}\right)} .
$$

As in [14, we use the shape parameter given by the cross-ratio

$$
\zeta:=\frac{\left(P_{i-1}-P_{i}\right)\left(P_{i+1}-P_{i+2}\right)}{\left(P_{i-1}-P_{i+1}\right)\left(P_{i}-P_{i+2}\right)},
$$

which gives the parameter associated to the edge $P_{i} P_{i+1}$. In the literature this $\zeta$ is often associated with the vertex ordering $P_{i}, P_{i+1}, P_{i+2}, P_{i-1}$.

Proof of Theorem 3.1. Start with $H_{1}$ positioned as $H_{\infty}$ and $H_{2}$ centered at 0 . Apply the isometry given by $M\left(H_{1}, H_{2}\right)$ which exchanges $H_{1}$ and $H_{2}$, so now $H_{2}$ is positioned at $H_{\infty}$ with $H_{1}$ centered at 0 . Next apply $M\left(H_{1}, H_{2}, H_{3}\right)^{-1}$ which translates $H_{2}$ to move $H_{3}$ to have center 0 . So now $H_{2}$ is positioned as $H_{\infty}$ and $H_{3}$ centered at 0 . Now repeat with $M\left(H_{2}, H_{3}\right)$ followed by $M\left(H_{2}, H_{3}, H_{4}\right)^{-1}$ to get $H_{3}$ positioned as $H_{\infty}$ and $H_{4}$ centered at 0 . After $n$ such steps we are back to the original positioning, so

$$
M\left(H_{n}, H_{1}, H_{2}\right)^{-1} M\left(H_{n}, H_{1}\right) \ldots M\left(H_{1}, H_{2}, H_{3}\right)^{-1} M\left(H_{1}, H_{2}\right)=I .
$$


Taking inverse of this equation (and keeping in mind that $M\left(H_{i}, H_{j}\right)$ is an involution) gives the desired result.

Proof of Theorem 3.2. Recall that indices are modulo $n$. We take $i=1$ and set $w=w\left(H_{1}, H_{2}\right), u^{\prime}=u\left(H_{0}, H_{1}, H_{2}\right), u=u\left(H_{1}, H_{2}, H_{3}\right)$, and we start with $H_{1}$ positioned as $H_{\infty}$ and $H_{2}$ centered at 0 as before. Then $H_{0}$ is centered at $-u^{\prime}$. Apply $M\left(H_{1}, H_{2}\right)$. Then the center of $H_{0}$ has been moved to $-w / u^{\prime}, H_{1}$ is centered at 0 , and $H_{2}$ is positioned as $H_{\infty}$. Now $H_{3}$ is centered at $u$. Taking the cross-ratio of $P_{0}=-w / u^{\prime}, P_{1}=0, P_{2}=\infty, P_{3}=u$ gives the result.

Theorem 3.3. The intercusp parameters $w\left(H_{i}, H_{j}\right)$ and the translation parameters $u\left(H_{i}, H_{j}, H_{k}\right)$ lie in the invariant trace field $k(X)$.

Proof. Let $\mathcal{P}$ be the set of all centers of marked horospheres which lift from cusp cross-sections of $X$. In [10, Theorem 2.4] it is shown that if three points of $\mathcal{P}$ are positioned at 0,1 and $\infty$, then $\mathcal{P}$ is positioned as subset of $k(X) \cup\{\infty\} \subset \mathbb{C} \cup\{\infty\}$.

To see $u\left(H_{i}, H_{j}, H_{k}\right) \in k(X)$ we position $H_{j}$ as $H_{\infty}$ and $H_{i}$ with center at 0 . Then there is also a marked horosphere centered at 1 so $\mathcal{P}$ is positioned as a subset of $k(X) \cup\{\infty\}$. So $u\left(H_{i}, H_{j}, H_{k}\right)=P_{k}-P_{i}=P_{k}$ is in $k(X)$.

Since shape parameters are also in $k(X)$, it now follows from Theorem 3.2 applied to a simplex with vertices $P_{h}, P_{i}, P_{j}, P_{k}$ that $w\left(H_{i}, H_{j}\right)$ is in $k(X)$.

If the image $\gamma_{i j}$ in $X$ of an intercusp geodesic arc $\gamma\left(H_{i}, H_{j}\right)$ is embedded, we call $\gamma_{i j}$ an intercusp arc of $X$ and if the line segment in an $H_{j}$ joining the endpoints of a $\gamma\left(H_{i}, H_{j}\right)$ and a $\gamma\left(H_{j}, H_{k}\right)$ has embedded image $\gamma_{i j k}$ in $X$, we call $\gamma_{i j k}$ a cusp arc of $X$.

Theorem 3.4. Suppose $Y \subset X$ is a union of cusp arcs and pairwise disjoint intercusp arcs, where any intercusp arcs which are not disjoint have been bent slightly near intersection points to make them disjoint, and suppose $\pi_{1}(Y) \rightarrow \pi_{1}(X)$ is surjective (equivalently, the lift $\tilde{Y} \in \mathbb{H}^{3}$ is connected). Then the intercusp and translation parameters corresponding to these arcs generate the invariant trace field.

Proof. If $k$ is a field, then the square of an element $\left(\begin{array}{ll}a & b \\ c & d\end{array}\right) \in \mathrm{PGL}_{2}(k)$ equals $\frac{1}{a d-b c}\left(\begin{array}{ll}a & b \\ c & d\end{array}\right)^{2} \in \mathrm{PSL}_{2}(k)$ and hence has PSL trace in $k$.

The conditions on $Y$ imply that each covering transformation in $\Gamma$ of the covering map $\mathbb{H}^{3} \rightarrow X$ is a product of matrices of the form $M\left(H_{i}, H_{j}\right)$ or $M\left(H_{i}, H_{j}, H_{k}\right)$. It is therefore in $P G L_{2}(k(X))$, so its square has PSL trace in $k(X)$. By [10, Theorem $2.1]$ the traces of squares of elements of $\Gamma$ generate the invariant trace field.

\section{Geometric applications}

4.1. Zickert's truncated triangulations. In [18 Christian Zickert considers an ideal triangulation of $X$ with the simplices truncated by removing horoballs centered at the vertices of the ideal simplices. He allows horoballs of any size, but we will use the ones normalized as in Section 2. He uses a labelling of these truncated simplices to give a particularly simple computation of the extended Bloch class and complex volume of $X$. The label $g_{i j}$ on a long edge of a truncated tetrahedron of the triangulation (see Figure 3i) is a matrix of the form $\left(\begin{array}{cc}0 & -\alpha^{-1} \\ \alpha & 0\end{array}\right)$ and a label 
$\alpha_{i k}^{j}$ on a short edge has the form $\left(\begin{array}{ll}1 & u \\ 0 & 1\end{array}\right)$. Note that $\left(\begin{array}{cc}0 & -\alpha^{-1} \\ \alpha & 0\end{array}\right)$ can be written as $\left(\begin{array}{cc}0 & -\alpha^{-2} \\ 1 & 0\end{array}\right) \in \mathrm{PGL}_{2}(\mathbb{C})$. It is not hard to check that $-\alpha^{2}$ represents the intercusp parameter $w\left(H_{i}, H_{j}\right)$ and $u$ represents the translation parameter $u\left(H_{i}, H_{j}, H_{k}\right)$, so with the horoballs chosen as in this paper, Zickert's parameters give elements of the form $\alpha^{2}$ and $u$ in $k(X)$.

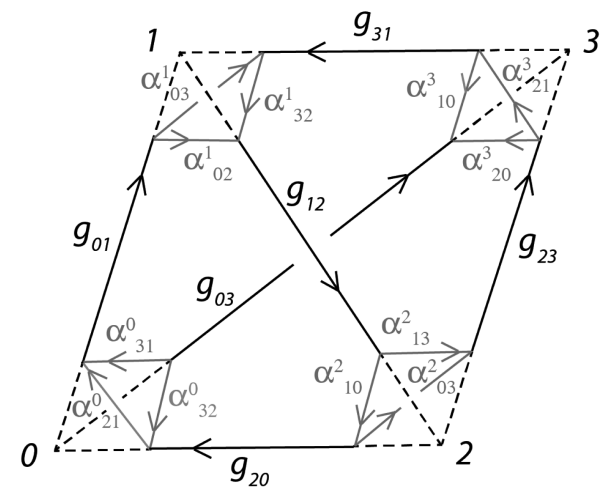

FiguRE 3. Zickert's truncated tetrahedron with labels.

\subsection{Parametrizing hyperbolic structure of link complements by complex}

labels. In [14, a new method for computing hyperbolic structure of links is suggested. It parametrizes horoball structure using complex labels, which then can be found from a link diagram that satisfies a few mild restrictions. The method is based on ideal polygons corresponding to the regions of a link diagram rather than decomposition of the complement into ideal tetrahedra. We will proceed by defining the labels; the method is described after that.

Suppose that $X$ is a link complement and that the link has a reduced diagram $D$ such that every arc from an overpass to an underpass of a crossing is properly homotopic to a geodesic in $X$. Conjecturally, every hyperbolic link admits such a diagram; for now it has been proved that every hyperbolic alternating link does (see 14 for a discussion and for the sufficient conditions on checkerboard surfaces). Existence of such a diagram for a link guarantees the applicability of the method.

The boundary of a $k$-sided region $R$ of the diagram $D$ is a union of $k \operatorname{arcs}$ on the boundary torus (we call them edges of $R$ ) and $k$ arcs, each of which goes from an overpass to an underpass of a crossing. Suppose $\Pi_{R}$ is a preimage of $R$ in $\mathbb{H}^{3}$. Then $\Pi_{R}$ is a cyclic sequence of $k$ segments of geodesics connecting the ideal points $P_{1}, \ldots, P_{k}$ in $\mathbb{H}^{3}$, and $k$ Euclidean segments on the corresponding marked horospheres $H_{1}, \ldots, H_{k}$ (see Figure 4).

Each geodesic $P_{i} P_{i+1}$ meets $H_{i}$ and $H_{i+1}$ in points $M_{i}$ and $N_{i+1}$ respectively. An orientation of the link determines a direction of the corresponding translation along the Euclidean line segment on $H_{i}$ in $\mathbb{H}^{3}$ joining $M_{i}$ with $N_{i}$. The corresponding translation parameter $\left(u\left(H_{i-1}, H_{i}, H_{i+1}\right)\right.$ or $u\left(H_{i+1}, H_{i}, H_{i-1}\right)=$ $-u\left(H_{i-1}, H_{i}, H_{i+1}\right)$, depending on the orientation) is called an edge label in 14 and is affixed to the side of the corresponding edge of $R$. A crossing label, affixed 


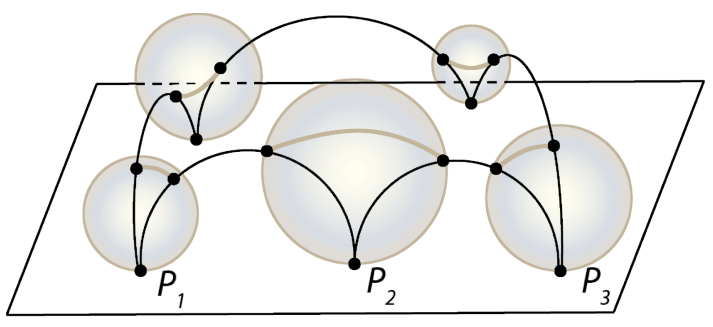

FiguRE 4. A preimage of the boundary of a 5-sided region of a link diagram.

to the crossing arc (or just to the corresponding crossing) that lifts to the geodesic $P_{i} P_{i+1}$, is the negative of the intercusp parameter $w\left(H_{i}, H_{i+1}\right)$.

A set of equations for edge and crossing labels, used to compute the hyperbolic structure on the link complement, is given in [14]. They consist of three equations resulting from the matrix relation equivalent to the one of Theorem 3.1 for each $\Pi_{R}$, and, for each arc between two crossings of the link diagram, an equation relating the values of the two edge labels corresponding to the regions on the two sides of the arc (for an alternating link the equation just says that the two labels differ by 1).

To summarize, instead of the traditional gluing and completeness relations based on shape parameters for an ideal triangulation, we now use two types of complex parameters: the intercusp parameter $w\left(H_{i}, H_{i+1}\right)$ describing distance and angle between two chosen cusps and the translation parameter $u\left(H_{j-1}, H_{j}, H_{j+1}\right)$ describing how the marked horospheres are situated with respect to each other. Theorem 3.3 showed that these parameters are elements of the invariant trace field. We have a finite number of such parameters describing the geometric structure of $X$, either using the labels that are assigned to a link diagram (edge and crossing labels) or labels assigned to a polyhedral decomposition (Zickert's parameters).

4.3. Generating the invariant trace field. It is not hard to see that Theorem 3.4 applies in both of the above cases, so we have:

Proposition 4.1. In each of the situations of subsections 4.1 and 4.2 the collection of intercusp and translation parameters described in that subsection generates the invariant trace field.

The number of parameters needed in the above proposition can be reduced as follows. A collection of intercusp arcs in $X$ is a tunnel collection if the arcs can be isotoped fixing their endpoints so that they are disjoint and the result of removing open horoball neighborhoods of the cusps and tubular neigborhoods of the arcs is a handlebody. The collections of intercusp arcs used in Proposition 4.1 is a tunnel collection, but usually a small subset of these intercusp arcs already is. For example, any two-bridge link has a tunnel collection consisting of a single intercusp arc.

Proposition 4.2. If $X$ has a tunnel collection consisting of $k$ intercusp arcs, then the invariant trace field can be generated by the $k$ intercusp parameters of these arcs together with $2 k$ translation parameters.

Proof. We number the cusps with $i=1, \ldots, h$. Suppose the $i$-th marked horospherical cusp section has $s_{i}$ endpoints of tunnel arcs on it. We can find a collection of 


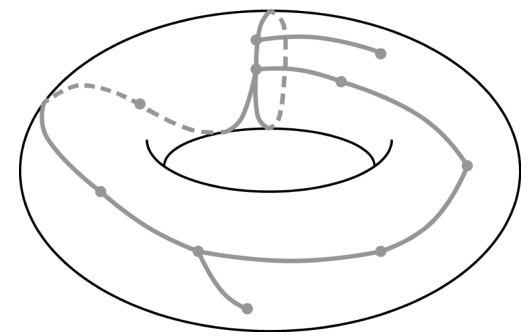

Figure 5. An example of a graph whose complement on the cusp section is an open disc.

$s_{i}+1$ cusp arcs connecting these endpoints such that their union is a graph whose complement on the cusp section is an open disc (as in Figure 5). The union of all these cusp arcs and isotoped tunnel arcs then satisfies Theorem 3.4 .

The total number of these cusp arcs is $2 k+h$. But the $s_{i}+1$ translation parameters at the $i$-th cusp section are linearly dependent modulo 1 , since a meridian of the cusp section has translation parameter 1 . We therefore only need $s_{i}$ translation parameters at the $i$-th cusp in applying Theorem 3.4 for a total of $2 k$ translation parameters.

In fact, usually a small subset of the intercusp and translation parameters generates the invariant trace field, even a small subset of the reduced collection of Proposition 4.2. For example, in [17] it is shown that for a 2-bridge link there is an ideal triangulation (in fact the canonical ideal triangulation) for which the simplex parameters are all rational functions of the crossing label $w_{1}$ of the leftmost crossing in the standard alternating diagram for the link (this crossing arc is a tunnel). By [10. Theorem 2.4] the simplex parameters of an ideal triangulation always generate the invariant trace field, so we get:

Proposition 4.3. For a two-bridge link the invariant trace field is generated by the single crossing label $w_{1}$ described above.

This proposition implies that for a hyperbolic 2-bridge link, the polynomial $P$ in $w_{1}$, obtained by applying the recursive process described in [17, has a zero which generates the invariant trace field.

Note that it is not guaranteed that $P$ is irreducible. In fact, suppose a reduced alternating diagram of a hyperbolic 2-bridge link has $k$ twists with $n_{1}, n_{2}, \ldots, n_{k}$ crossings. The calculation of [17] gives an upper bound $m_{1}^{3} m_{2}^{3} \ldots m_{k}^{3}$ for the degree of the polynomial $P$, where $m_{i}=n_{i}$ if $n_{i}>1$, and $m_{i}=2$ otherwise. The work of Riley [13] provides a sharper upper bound $(\alpha-1) / 2$ for the degree of the invariant trace field, where $(\alpha, \beta)$ denotes the normal form of the 2-bridge type, given by $\left.\alpha / \beta=m_{1}+1 /\left(m_{2}+1 /\left(\cdots+1 / m_{k}\right)\right) \ldots\right)$. Experiment shows that Riley's bound is often sharp.

Remark 4.4. Another example is the infinite family of links that are closures of the braid $\left(\sigma_{1} \sigma_{2}^{-1}\right)^{n}$. Symmetry allows us to use just three diagram labels, and a quick computation then shows that just one translation parameter suffices and the invariant trace field is generated over $\mathbb{Q}$ by $\sqrt{-3-4 \cos (\pi / n)+4 \cos ^{2}(\pi / n)}$ (see the "Examples" section of [14 for a picture and relations). 
In fact, for a "random" knot chosen from existing knot tables it is rare that the invariant trace field is not generated by a single one of the parameters, since it is unusual that the invariant trace field has a proper subfield of degree $>1$.

\section{ACKNOWLEDGMENTS:}

The authors would like to thank Morwen Thistlethwaite for help with computational aspects, and Alan Reid for bringing attention to the rep-polynomial in the work of Riley. The authors also acknowledge support from U.S. National Science Foundation grants DMS 1406588, 1107452, 1107263, 1107367 "RNMS: GEometric structures And Representation varieties" (the GEAR Network).

\section{REFERENCES}

[1] Colin C. Adams, Waist size for cusps in hyperbolic 3-manifolds, Topology 41 (2002), no. 2, 257-270, DOI 10.1016/S0040-9383(00)00034-3. MR1876890 (2003e:57023)

[2] A. Borel, Commensurability classes and volumes of hyperbolic 3-manifolds, Ann. Scuola Norm. Sup. Pisa Cl. Sci. (4) 8 (1981), no. 1, 1-33. MR616899 (82j:22008)

[3] David Coulson, Oliver A. Goodman, Craig D. Hodgson, and Walter D. Neumann, Computing arithmetic invariants of 3-manifolds, Experiment. Math. 9 (2000), no. 1, 127-152. MR.1758805 (2001c:57014)

[4] D. B. A. Epstein and R. C. Penner, Euclidean decompositions of noncompact hyperbolic manifolds, J. Differential Geom. 27 (1988), no. 1, 67-80. MR918457(89a:57020)

[5] Hugh M. Hilden, María Teresa Lozano, and José María Montesinos-Amilibia, A characterization of arithmetic subgroups of $\mathrm{SL}(2, \mathbf{R})$ and $\mathrm{SL}(2, \mathbf{C})$, Math. Nachr. 159 (1992), 245-270, DOI 10.1002/mana.19921590117. MR.1237113 (94i:20088)

[6] Melissa L. Macasieb, Kathleen L. Petersen, and Ronald M. van Luijk, On character varieties of two-bridge knot groups, Proc. Lond. Math. Soc. (3) 103 (2011), no. 3, 473-507, DOI 10.1112/plms/pdr003. MR2827003 (2012j:57015)

[7] Colin Maclachlan and Alan W. Reid, The arithmetic of hyperbolic 3-manifolds, Graduate Texts in Mathematics, vol. 219, Springer-Verlag, New York, 2003. MR1937957 (2004i:57021)

[8] William W. Menasco, Polyhedra representation of link complements, Low-dimensional topology (San Francisco, Calif., 1981), Contemp. Math., vol. 20, Amer. Math. Soc., Providence, RI, 1983, pp. 305-325, DOI 10.1090/conm/020/718149. MR718149 (85e:57006)

[9] G. D. Mostow, Quasi-conformal mappings in $n$-space and the rigidity of hyperbolic space forms, Inst. Hautes Études Sci. Publ. Math. 34 (1968), 53-104. MR0236383 (38 \#4679)

[10] Walter D. Neumann and Alan W. Reid, Arithmetic of hyperbolic manifolds, Topology '90 (Columbus, OH, 1990), Ohio State Univ. Math. Res. Inst. Publ., vol. 1, de Gruyter, Berlin, 1992, pp. 273-310. MR.1184416 (94c:57024)

[11] Gopal Prasad, Strong rigidity of Q-rank 1 lattices, Invent. Math. 21 (1973), 255-286. MR0385005 (52 \#5875)

[12] Alan W. Reid, A note on trace-fields of Kleinian groups, Bull. London Math. Soc. 22 (1990), no. 4, 349-352, DOI 10.1112/blms/22.4.349. MR1058310 (91d:20056)

[13] Robert Riley, Parabolic representations of knot groups. I, Proc. London Math. Soc. (3) 24 (1972), 217-242. MR0300267 (45 \#9313)

[14] Morwen Thistlethwaite and Anastasiia Tsvietkova, An alternative approach to hyperbolic structures on link complements, Algebr. Geom. Topol. 14 (2014), no. 3, 1307-1337, DOI 10.2140/agt.2014.14.1307. MR.3190595

[15] W. P. Thurston, The Geometry and Topology of Three-Manifolds, Electronic Version 1.1 (March 2002), http://www.msri.org/publications/books/gt3m/

[16] A. Tsvietkova, Hyperbolic links complements, Ph.D. Thesis, University of Tennessee, 2012.

[17] Anastasiia Tsvietkova, Exact volume of hyperbolic 2-bridge links, Comm. Anal. Geom. 22 (2014), no. 5, 881-896. MR 3274953 
[18] Christian K. Zickert, The volume and Chern-Simons invariant of a representation, Duke Math. J. 150 (2009), no. 3, 489-532, DOI 10.1215/00127094-2009-058. MR2582103 (2011c:58053)

Department of Mathematics, Barnard College, Columbia University, 2990 Broadway MC4429, New York, New York 10027

E-mail address: neumann@math.columbia.edu

Department of Mathematics, University of California - Davis, One Shields Ave, Davis, California 95616

E-mail address: tsvietkova@math.ucdavis.edu 